\title{
Pediatric longitudinal melanonychia
}

\author{
Angela Burleigh MD PhD, Joseph M. Lam MD
}

Cite as: CMAJ 2017 August 28;189:E1093. doi: 10.1503/cmaj.170256

A 9-year-old boy of Asian descent was referred to the outpatient pediatric dermatology clinic with an asymptomatic linear brown discolouration affecting his left middle fingernail (Figure 1A). This discolouration had been present for about one year and had not changed in thickness or pigment intensity. The patient was otherwise healthy and was not taking any medications. There was no personal or family history of melanoma.

Upon physical examination, there was a linear band of brown pigment extending from the eponychium to the free edge of the otherwise smooth nail plate (Figure 1A). Dermoscopy (Figure 1B) showed a homogeneous linear pigmentation that was visible through the translucent cuticle and proximal nail fold (pseudoHutchinson sign). This is distinguished from Hutchinson sign, in which the extension of brown-black pigment onto the adjacent cuticle and nail folds is suggestive of subungual melanoma. We diagnosed longitudinal melanonychia (or melanonychia striata).

Longitudinal melanonychia is the presence of a pigmented longitudinal streak along the nail plate from increased melanin production and deposition within the nail plate. Prevalence ranges from $1.4 \%$ in white populations to $77 \%$ in black populations. In some populations, multiple nails are affected occasionally. ${ }^{1,2}$ In children, longitudinal melanonychia most commonly occurs because of an underlying benign melanocytic nevus (beneath the proximal nail fold), lentigo or melanocyte activation within the nail matrix; ${ }^{3}$ subungual melanoma is uncommon in this population. ${ }^{4}$

Diagnostic biopsy should be avoided where possible, given the likelihood of permanent nail dystrophy. ${ }^{5}$ Features that should prompt histopathologic examination include increasing width or darkening of the lesion, pigment variegation, periungual pigmentation or dystrophy of the nail plate. ${ }^{3,4}$ Clinicians should advise parents of pediatric patients to monitor lesions closely for any of these described changes and present for prompt evaluation should they occur. Prolonged follow-up is advised, given that melanoma of the nail apparatus in adults with a history of longitudinal melanonychia beginning in childhood has been reported on occasion. ${ }^{3}$

\section{References}

1. Duhard E, Calvet C, Mariotte N, et al. Prevalence of longitudinal melanonychia in the white population [article in French]. Ann Dermatol Venereol 1995;122:586-90.

2. Leyden JJ, Spott DA, Goldschmidt H. Diffuse and banded melanin pigmentation in nails. Arch Dermatol 1972;105:548-50.

3. Cooper C, Arva NC, Lee C, et al. A clinical, histopathologic, and outcome study of melanonychia striata in childhood. J Am Acad Dermatol 2015;72:773-9.

4. Antonovich DD, Grin C, Grant-Kels JM. Childhood subungual melanoma in situ in diffuse nail melanosis beginning as expanding longitudinal melanonychia. Pediatr Dermatol 2005;22:210-2.

5. Grover C, Nanda S, Reddy BS, et al. Nail biopsy: assessment of indications and outcome. Dermatol Surg 2005;31:190-4.

Competing interests: None declared.

This article has been peer reviewed.

The authors have obtained patient consent.

Affiliations: Departments of Dermatology and Skin Science (Burleigh), Pediatrics (Lam) and Dermatology (Lam), Faculty of Medicine, The University of British Columbia, Vancouver, BC

Correspondence to: Angela Burleigh, aburleigh@me.com 